\title{
VIENT DE PARAÎTRE
}

CAWS, PETER. Structuralism. New Jersey and London: Humanities Press International, Inc. 1991 (Second Paperback Printing).

DENEYS-TUNNEY. Ecriture du corps: de Descartes a Laclos. Paris: Presses Universitaires de France, 1992.

DUMOULIe, CAMILIE Nietzsche et Artaud: Pour une éthique de la cruaute. Paris: Presses Universitaires de France, 1992.

HUSSERL,EDMUND. Problèmes fondamentauxde la phénoménologie. Traduction, indications, notes, remarques et index par Jacques English. Paris: Presses Universitaires de France, 1991.

IMBERT, CLAUDE Phénoménologies et langues formulaires. Paris: Presses Universitaires de France, 1992.

MaBRIDE, WILIAM L Sartre's Political Theory. Bloomington and Indianapolis: Indiana University Press, 1991.

MOLNAR, THOMAS. Philosphical Grounds. New York, Paris, London: Peter Lang, 1991.

SILVERMAN, HUGH J. and BARRY, JAMES, Jr. Eds. Texts and Dialogues: Merleau-Ponty. New Jersey \& London: Humanities Press, 1992. 